\title{
Estudo dos instrumentos de avaliação funcional em demência comumente utilizados no Brasil**
}

\author{
Study of functional assessment tools in dementia \\ commonly used in Brazil
}

\author{
Mariana Boaro Fernandez Canon ${ }^{1}$, Marcia Maria P. Camargo Novelli²
}

CANON, M. B. F.; NOVELLI, M. M. P. C. Estudo dos instrumentos de avaliação funcional em demência comumente utilizados no BrasiL. Rev. Ter. Ocup. Univ. São Paulo, v. 23, n. 3, p. 253-262, set./dez. 2012.

RESUMO: Esta pesquisa objetivou identificar os instrumentos de avaliação funcional utilizados em demência no Brasil. Foram acessados os bancos de dados LILACS, PUBMED, SciELO e Portal Periódicos CAPES para o levantamento de estudos que utilizaram instrumentos de avaliação funcional em demência. Os instrumentos identificados foram divididos por grupos de atividades avaliadas, finalidade de uso e identificado se os mesmos haviam passado pelo processo de adaptação transcultural e análise da confiabilidade e validade. Foram selecionados 46 artigos e 20 teses, que utilizaram em seus estudos instrumentos de avaliação funcional. Quinze instrumentos foram identificados: 4 avaliam Atividades Instrumentais da Vida Diária (AIVD), 3 Atividades Básicas da Vida Diária (ABVD), 4 ABVD e AIVD, 3 ABVD, AIVD e Lazer e 1 ABVD e Lazer, evidenciando a falta de instrumentos que avaliem o grupo de atividades de trabalho, assim como a falta de uma avaliação que contemple todos os grupos de atividades. Dos instrumentos identificados, 12 passaram pelo processo de adaptação transcultural, 9 apresentavam dados de confiabilidade e 5 de validade, sendo 4 para uso em demência. Por finalidade de uso 5 instrumentos eram avaliativos e 10 descritivos. Este estudo identificou que os instrumentos de avaliação funcional em demência, comumente utilizados no Brasil, tem sua origem em outros países, constatando a falta de produções nacionais, além disso, a avaliação das propriedades psicométricas não está sendo realizada para todos os instrumentos adaptados a nossa cultura.

DESCRITORES: Idosos; Demência; Avaliação geriátrica.

\footnotetext{
* Este trabalho apresenta os resultados obtidos em um projeto de Iniciação Científica, financiando pela Fundação de Amparo à Pesquisa do Estado de São Paulo (FAPESP - 2008/09717-7). Apresentado no XVIII Congresso de Iniciação Científica - PIBIC - UNIFESP, São Paulo, SP, 23 jun. 2010 (apresentação oral), I Simpósio Internacional de Pesquisa em Terapia Ocupacional, São Paulo, SP, $27-28$ ago. 2010 (apresentação de pôster).

'. Terapeuta Ocupacional, Mestranda em Ciências da Saúde pela Universidade Federal de São Paulo - Campus Baixada Santista; E-mail: mcanon.to@gmail.com.

2. Professor Adjunto Curso de Terapia Ocupacional da Universidade Federal de São Paulo - Campus Baixada Santista; E-mail: mnovelli@ uol.com.br.
}

Endereço para correspondência: Mariana Boaro F. Canon. Avenida Jurema, nº 38, Apto. 21, Moema, São Paulo, SP, CEP: 04079-000. 


\section{INTRODUÇÃO}

$\mathrm{O}$ século XX foi marcado por uma revolução da longevidade, que continua nos dias de hoje tomando rumos ainda maiores, visto que as pessoas acima de 65 anos em 2010 corresponderam a 7,4\% da população total. No entanto a melhora na qualidade de vida não está acompanhando esta revolução, impedindo assim que as pessoas tenham um envelhecimento bem sucedido (NETTO, 2007; IBGE, 2010).

Isto acarreta aumento do número de casos de doenças crônico-degenerativas e limitações funcionais, representando para a sociedade e para os órgãos governamentais desafios médicos e socioeconômicos próprios do envelhecimento populacional (NETTO, 2007).

Entre as doenças crônico-degenerativas, destacamse as demências, que segundo o DSM-IV-TR, são caracterizadas pelo declínio cognitivo persistente, sendo que a principal função comprometida é a memória além de outras funções, que interfere de modo significativo no funcionamento ocupacional do indivíduo, visto que a progressão da doença acarreta no comprometendo do desempenho nas atividades cotidianas, consequentemente gerando incapacidades e dependência funcional (APA, 2000; VASCONCELOS et al., 2007).

Entre os grupos de atividades desempenhadas pelos indivíduos temos as atividades básicas da vida diária (ABVD) que por definição são aquelas relacionadas ao cuidado do indivíduo consigo mesmo e incluem tarefas como: higiene pessoal e autocuidado, banho, alimentação, vestuário, entre outras. E ainda as atividades instrumentais da vida diária (AIVD) que relacionam o indivíduo com o meio e por isso são complexas e incluem, compras, limpeza, uso de equipamentos para comunicação, mobilidade na comunidade, entre outras (MELLO; MANCINI, 2007).

O grupo de atividades de trabalho é representado pelas atividades produtivas, sendo estas remuneradas ou não. E por último o grupo de atividades de lazer, que por definição são as atividades que realizamos por prazer e que para cada indivíduo podem ser representadas por diversas atividades (MELLO; MANCINI, 2007).

A capacidade funcional pode ser compreendida como o potencial que os indivíduos apresentam de realizar essas e outras atividades de forma independente. Pode ser avaliada através de instrumentos de avaliação funcional padronizados, que descrevem de forma ordenada o desempenho nestas atividades que são típicas do cotidiano de um indivíduo, e pressupõem uma variedade de habilidades (MELLO; MANCINI, 2007).
Como a maioria dos instrumentos tem origem em outros países, e consequentemente foram produzidos em outros idiomas, um aspecto a ser considerado é se o instrumento traduzido para o português passou pelo processo de adaptação transcultural para a seguir submetê-los a análise de suas propriedades psicométricas (NOVELLI et al., 2010).

O Scientific Advisory Committee of Medical Outcomes Trust (SAC) propõem a utilização de critérios para revisão de instrumentos de avaliação (SAC, 2002).

Esses critérios nos permitem avaliar as propriedades psicométricas dos instrumentos e os atributos a serem considerados no processo de escolha dos mesmos. As propriedades psicométricas fundamentais propostas são: confiabilidade e validade (NOVELLI et al., 2010).

A confiabilidade analisa a consistência interna e a capacidade de reprodutibilidade do instrumento ao longo tempo, sendo que um instrumento é considerado confiável quando um escore similar é conseguido na administração repetida (POLGAR, 2002).

A validade é a propriedade mais importante a se considerar quando analisamos um teste, ela avalia o grau com que o instrumento mede o que se propõe a medir, verificando assim a coerência do conteúdo (POLGAR, 2002).

Outro aspecto relevante, na análise de instrumentos de avaliação, é classificar os mesmos com relação a sua finalidade de uso, podendo ser descritivos, avaliativos e preditivos (TEDESCO, 2002).

Em uma avaliação descritiva busca-se uma visão objetiva do indivíduo no contexto atual, preferencialmente é obtida por meio de aplicação rápida e normalmente é utilizada para. No processo avaliativo procura-se avaliar as mudanças ao longo do tempo (TEDESCO, 2002).

As avaliações preditivas baseiam-se em uma probabilidade de como o indivíduo estará em uma dada situação no futuro. Porém, o desempenho funcional de uma variável pode ser modificado constantemente devido à muitas alterações biopsicossociais, sendo assim, as avaliações com finalidade preditiva não são encontradas entre os instrumentos de avaliação funcional (TEDESCO, 2002).

Para a avaliação do desempenho em atividades que compõe o cotidiano de um indivíduo, faz-se necessário a busca de instrumentos de avaliação confiáveis e válidos, uma vez que contribuem para: o diagnóstico clínico e terapêutico ocupacional; para a avaliação e acompanhamento do desempenho funcional dos pacientes e para fornecer 
subsídios às intervenções de Terapia Ocupacional.

O presente estudo tem como objetivo apresentar os resultados de um levantamento de instrumentos de avaliação funcional em demência, comumente utilizados no Brasil, classificando-os quanto aos grupos de atividades, finalidade de uso e quanto à submissão ao processo de adaptação transcultural e avaliação das propriedades psicométricas (confiabilidade e validade).

\section{METODOLOGIA}

A primeira etapa consistiu no levantamento de artigos nos bancos de dados LILACS, PUBMED, Scielo e Portal Periódicos CAPES (banco de teses), entre os meses de março de 2009 e janeiro de 2010. As palavras chave foram selecionadas com base nos grupos de atividades e estão descritas nos Quadros 1 e 2.

Quadro 1 - Palavras chave utilizadas para o levantamento dos estudos nos bancos de dados.
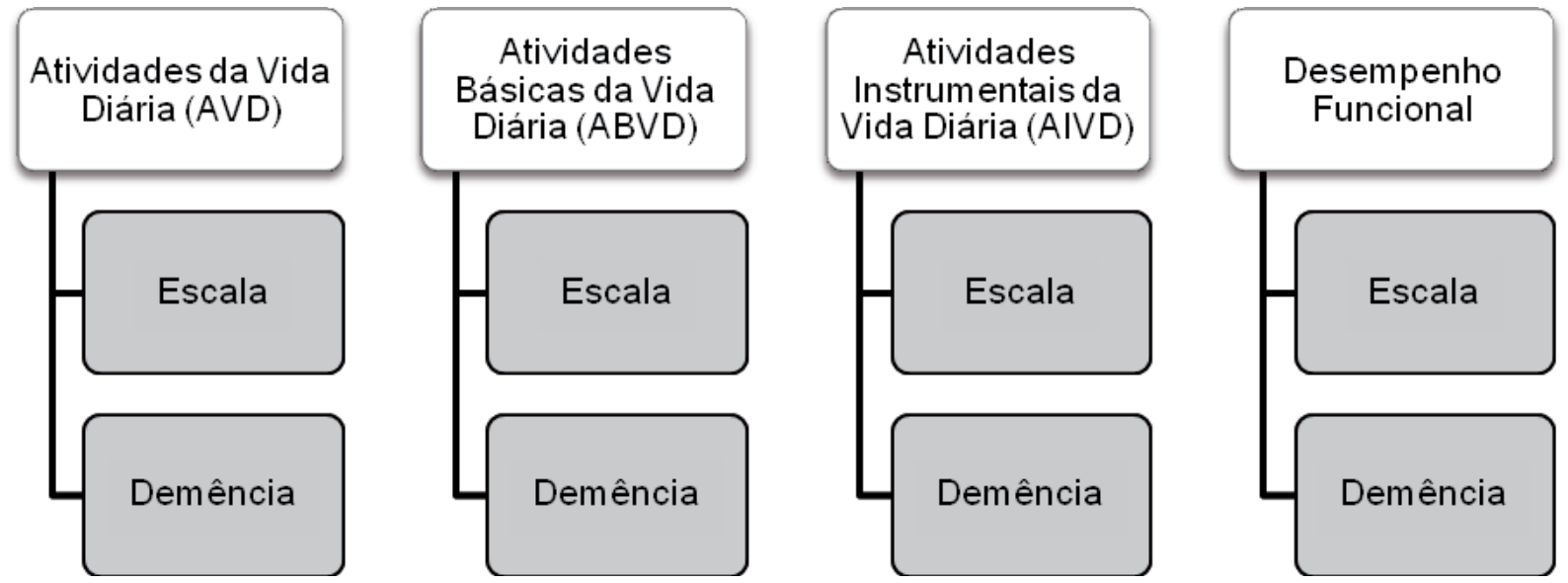

Quadro 2 - Palavras chave utilizadas, em inglês, para o levantamento dos estudos nos bancos de dados.
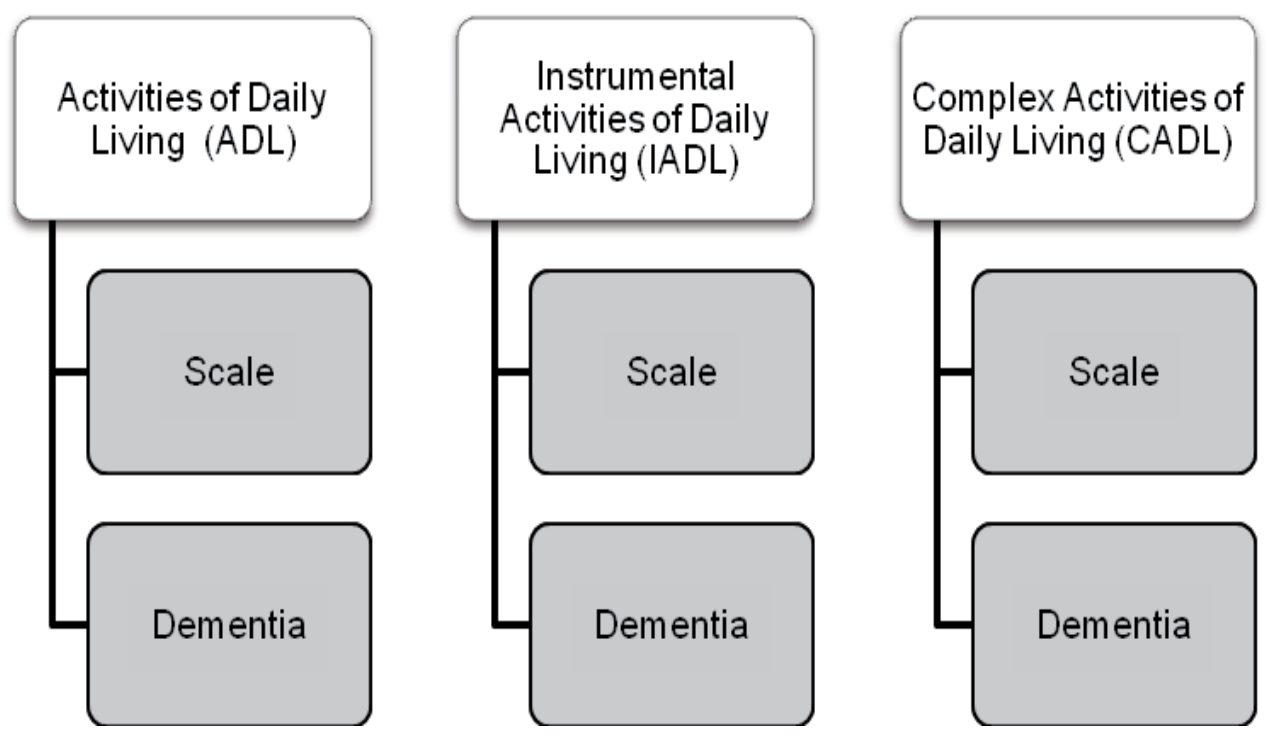
Os estudos selecionados deveriam ser de autores brasileiros, conterem resumo em português e/ou inglês, constar no resumo a população abordada, devendo esta ser de idosos com demência e constar os instrumentos de avaliação funcional utilizados, ou mencionar o uso destes.

Após a seleção dos artigos e teses, a pesquisa seguiu no levantamento dos instrumentos de avaliação funcional, buscando confirmar a relação destes com demência e informações sobre o processo de adaptação transcultural e de avaliação de suas propriedades psicométricas, através de análise qualitativa.

O estudo considerou como uma das medidas de confiabilidade a indicação de consistência interna pelo coeficiente alpha de Cronbach. Esse coeficiente pode variar de 0 a 1,0 . O SAC aponta que valores de 0 a 0,6 indicam confiabilidade insatisfatória; de 0,61 a 0,7 é satisfatória e de 0,71 a 1,0 a confiabilidade é elevada (SAC, 1995).

Para análise de confiabilidade inter e intra examinador o estudo considerou os valores dos índices de Kappa. Para sua interpretação foram utilizados os critérios propostos por Landis e Koch (1977), sendo eles: de 0,81 a 1,0 para a confiabilidade quase perfeita; 0,61 a 0,8 boa confiabilidade; 0,41 a 0,6 confiabilidade moderada; 0,21 a 0,4 regular; 0,1 a 0,2 discreta e 0,0 a 0,1 pobre.

Em seguida estes instrumentos foram analisados qualitativamente e divididos pelos grupos de atividades que se propõe a avaliar (ABVD, AIVD, Lazer e Trabalho) e por finalidade de uso (descritivos e avaliativos).

\section{RESULTADOS}

Foram selecionados 51 artigos no banco de dados PUBMED, 48 no LILACS, 33 no SciELo e 49 teses no Portal de Periódicos da CAPES, totalizando 181 estudos, porém desse total, muitos artigos e teses eram duplicados, finalizando a seleção com 46 artigos e 20 teses, apresentando, aproximadamente, $63,5 \%$ de duplicidade.

Todos os instrumentos selecionados foram acessados, através dos anexos dos artigos/teses e solicitação aos autores, totalizando 15 instrumentos de avaliação funcional em demência.

A Tabela 1 apresenta a classificação dos instrumentos selecionados, com relação aos grupos de atividades.

Tabela 1 - Classificação dos instrumentos de avaliação funcional em Demência, com relação aos grupos de atividades

\begin{tabular}{lc} 
Grupos de atividades avaliados & Total de Instrumentos (\%) \\
\hline ABVD & $3(20)$ \\
AIVD & $4(26,6)$ \\
ABVD e AIVD & $4(26,6)$ \\
ABVD, AIVD e Lazer & $3(20)$ \\
ABVD e Lazer & $1(6,6)$ \\
Total & $15(100)$ \\
\hline
\end{tabular}

De 100\% (15) dos instrumentos selecionados, aproximadamente $73,3 \%$ (11) dos instrumentos avaliam ABVD, 73,3\% (11) avaliam AIVD, 26,6\% (4) lazer e não foi identificado nenhum instrumento que avaliasse o grupo de atividades de trabalho. Dos instrumentos identificados $20 \%$ (3) avaliam mais de um grupo de atividades, como ABVD, AIVD e lazer e 26,6\% (4) avaliam ABVD e AIVD.

Com relação à finalidade de uso, verificou-se que 5 instrumentos são avaliativos e 10 descritivos.
São descritas, nas Tabelas 2 e 3, algumas informações sobre os instrumentos de avaliação funcional selecionados neste estudo.

Destaca-se que alguns instrumentos de avaliação funcional não apresentam estudo nacional ou brasileiro, sobre a adaptação transcultural e a avaliação das propriedades psicométricas, porém estes permaneceram no levantamento desta pesquisa, visto que esse não era um item de exclusão. 
CANON, M. B. F. et al. Estudo dos instrumentos Rev. Ter. Ocup. Univ. São Paulo, v. 23, n. 3, p. 253-62, set./dez. 2012.

Tabela 2 - Instrumentos de avaliação funcional em demência, classificados em relação aos grupos de atividade, critérios de revisão do $\mathrm{SAC}$ e finalidade de uso

\begin{tabular}{|c|c|c|c|}
\hline Instrumentos de Avaliação Funcional & $\begin{array}{l}\text { Grupos de } \\
\text { Atividades }\end{array}$ & Critérios de Revisão do SAC & Finalidade de Uso \\
\hline $\begin{array}{l}\text { Escala de Independência em Atividades da Vida Diária } \\
\text { (Escala de Katz) }\end{array}$ & ABVD & $\begin{array}{l}\text { Adaptado; Dados de } \\
\text { Confiabilidade }\end{array}$ & Descritivo \\
\hline Medida de Independência Funcional (MIF) & ABVD & $\begin{array}{l}\text { Adaptado; Dados de } \\
\text { Confiabilidade; Validado }\end{array}$ & Avaliativo \\
\hline Southampton Assessment of Mobility (SAM) & ABVD & $\begin{array}{l}\text { Adaptado; Dados de } \\
\text { Confiabilidade }\end{array}$ & Descritivo \\
\hline Questionário de Atividades Instrumentais de Pfeffer & AIVD & + & Descritivo \\
\hline $\begin{array}{l}\text { Escala de Atividades Instrumentais da Vida Diária } \\
\text { (EAIVD - Lawton and Brody) }\end{array}$ & AIVD & $\begin{array}{l}\text { Adaptado; Dados de } \\
\text { Confiabilidade }\end{array}$ & Descritivo \\
\hline $\begin{array}{l}\text { Informant Questionnaire on Cognitive Decline in the } \\
\text { Elderly (IQCODE) }\end{array}$ & AIVD & $\begin{array}{l}\text { Adaptado; Dados de } \\
\text { Confiabilidade }\end{array}$ & Descritivo \\
\hline Teste Funcional (Ávila, 2004) & AIVD & & Descritivo \\
\hline $\begin{array}{l}\text { Direct Assessment of Functional Status Test (DAFS- } \\
\text { BR) }\end{array}$ & ABVD e AIVD & $\begin{array}{l}\text { Adaptado; Dados de } \\
\text { Confiabilidade; Validado }\end{array}$ & Avaliativo \\
\hline Blessed Dementia Rating Scale (BDRS) & ABVD e AIVD & - & Descritivo \\
\hline $\begin{array}{l}\text { Alzheimer 's Disease Cooperative Study - Clinical } \\
\text { Global Impression of Change (ADCS-CGIC) }\end{array}$ & ABVD e AIVD & Adaptado & Avaliativo \\
\hline Activities of Daily Living Questionnaire (ADLQ) & ABVD e AIVD & $\begin{array}{l}\text { Adaptado; Dados de } \\
\text { Confiabilidade; Validado }\end{array}$ & Descritivo \\
\hline Escala Bayer de Atividades da Vida Diária (B-ADL) & $\begin{array}{l}\text { ABVD, AIVD e } \\
\text { Lazer }\end{array}$ & Adaptado & Descritivo \\
\hline $\begin{array}{l}\text { Escala de Avaliação de Incapacidade em Demência } \\
\text { (DAD) }\end{array}$ & $\begin{array}{l}\text { ABVD, AIVD e } \\
\text { Lazer }\end{array}$ & $\begin{array}{l}\text { Adaptado; Dados de } \\
\text { Confiabilidade; Validado }\end{array}$ & Avaliativo \\
\hline Bristol Daily Activities Functional Scale & $\begin{array}{l}\text { ABVD, AIVD e } \\
\text { Lazer }\end{array}$ & $\longrightarrow$ & Avaliativo \\
\hline Clinical Dementia Rating Scale (CDR) & ABVD e Lazer & $\begin{array}{l}\text { Adaptado; Dados de } \\
\text { Confiabilidade; Validado }\end{array}$ & Descritivo \\
\hline
\end{tabular}


CANON, M. B. F. et al. Estudo dos instrumentos Rev. Ter. Ocup. Univ. São Paulo, v. 23, n. 3, p. 253-62, set./dez. 2012.

Tabela 3 - Características dos instrumentos de avaliação funcional em demência comumente utilizados no Brasil

\begin{tabular}{|c|c|c|}
\hline $\begin{array}{l}\text { Instrumentos de Avaliação } \\
\text { Funcional }\end{array}$ & Itens & Tipo de análise - Índices de Confiabilidade \\
\hline KATZ & 6 & 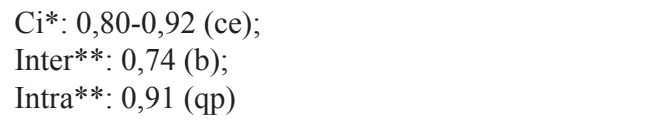 \\
\hline SAM & 4 & $\begin{array}{l}\text { Inter: DL: 0,89 (qp), DM: 0,79 (b) e DG: 0,53 (m); } \\
\text { Intra: DL: 0,86 (qp), DM: 0,85 (qp) e DG: 0,49 (m) }\end{array}$ \\
\hline MIF & 6 & Inter: 0,87-0,98 (qp); Intra: 0,91-0,98 (qp) \\
\hline PFQA & 10 & 0 \\
\hline EAIVD & 7 & $\begin{array}{l}\text { Intra: Reprod. 0,89 (qp) } \\
\text { Objt. } 0,80 \text { (qp) }\end{array}$ \\
\hline IQCODE & 26 & $\begin{array}{l}\text { Ci: } 0,94(c e) \\
\text { Intra: } 0,92(q p)\end{array}$ \\
\hline TESTE FUNC. & 3 & 0 \\
\hline DAFS-BR & 6 & $\begin{array}{l}\text { Ci: } 0,78 \text { (ce); } \\
\text { Inter: } 0,99 \text { (qp) }\end{array}$ \\
\hline BDRS & 22 & 0 \\
\hline ADCS-CGIG & 4 & 0 \\
\hline ADLQ & 6 & Ci: 0,759 (ce) \\
\hline B-ADL & 25 & 0 \\
\hline DAD & 10 & $\begin{array}{l}\text { Inter: } 0,72(b) \\
\text { Intra: } 0,85 \text { (qp) }\end{array}$ \\
\hline BRISTOL & 20 & 0 \\
\hline CDR & 6 & Inter: $0,73(b)$ \\
\hline
\end{tabular}

*Consistência interna (expressa pelo Alpha de Cronbach - ce: confiabilidade elevada).

**Confiabilidade Intra examinador e Inter examinador (expressa pelo Kappa - qp: confiabilidade quase perfeita; b: confiabilidade boa e m: confiabilidade moderada).

DL: Demência Leve; DM: Demência Moderada e DG: Demência Grave.

\section{DISCUSSÃO}

A tradução de instrumentos em outras línguas vem substituindo a criação de novos. Atualmente, um dos aspectos considerados pelos editores na criação de um novo teste, tem sido a possibilidade da tradução deste para outros idiomas, ou ainda a criação de instrumentos na proposição transcultural (GIUSTI; BEFI-LOPES, 2008).
Os atributos propostos pelo SAC são abordados como tema central de alguns artigos, demonstrando a importância de sua análise e que certos instrumentos de avaliação funcional podem ser utilizados com segurança em nosso país, pois estão de acordo com nossa cultura e idioma.

A confiabilidade e a validade são atributos fundamentais para a escolha de um instrumento, uma vez que comprovam a capacidade do instrumento de medir o 
que se propõe e a confiabilidade das informações advindas do mesmo.

Aqueles que não possuem esses atributos podem ocasionar o levantamento de informações não fidedignas para a definição das condutas terapêuticas, elaboração do plano de intervenção, chegando a comprometer a eficiência dos tratamentos oferecidos (GIUSTI; BEFI-LOPES, 2008).

Outro aspecto a ser considerado quando são utilizados instrumentos produzidos em outra língua, é a necessidade de estudos de normatização das notas de corte dos instrumentos adaptados, que podem sofrer alterações relacionadas à cultura alvo da aplicação. $\mathrm{Na}$ análise dos instrumentos identificados apenas no estudo realizado com a Escala Katz, os autores descrevem esse processo de normatização das notes de corte.

A pontuação é tão importante quanto os itens a serem avaliados, isso porque, através dela podemos classificar os indivíduos em relação ao seu desempenho funcional, e da mesma forma que as atividades avaliadas devem ser adaptadas ao contexto de cada população, a pontuação deve, também, estar coerente a cada cultura.

Um aspecto a ser ressaltado é a falta de estudos de validação para os instrumentos já adaptados a nossa cultura, pois apenas 26,6\% (4) são validados para o uso em demência no nosso país.

Outra questão importante identificada foram os grupos de atividades que cada instrumento se propõe a avaliar, como ABVD, AIVD, lazer e trabalho. É importante destacar que para se apreender a dimensão completa de um grupo de atividades, o instrumento deve conter vários itens pertinentes àquele grupo, garantindo um mapeamento mais completo.

O grupo de atividades lazer é avaliado em 4 instrumentos identificados, que contem apenas um item direcionado a esse assunto, no qual é perguntado se o paciente realizou/realiza atividades de lazer.

O construto de lazer precisa ser melhor identificado em nossa cultura, assim como as atividades comuns a este grupo, para que itens possam ser inseridos e o construto devidamente avaliado nos instrumentos.

As atividades produtivas não são avaliadas em nenhum instrumento de avaliação funcional, e a justificativa para isso não é apresentada em nenhum estudo sobre desempenho funcional.

Há idosos em estágios iniciais de demência ou mesmo com Comprometimento Cognitivo Leve que estão inseridos no mercado de trabalho, apontando a importância deste item e a sua inserção nas escalas de avaliação, visto que qualquer alteração no desempenho funcional em relação às atividades produtivas é de extrema relevância tanto para o diagnóstico quanto para o tratamento.

Um novo grupo de atividades foi proposto na literatura, Atividades Avançadas da Vida Diária (AAVD), que são consideradas de alta complexidade. Incluem: dirigir automóveis, andar de bicicleta, tocar um instrumento musical, viajar, participar de serviço comunitário e de atividades políticas. Esse grupo de atividades não é considerado essencial para definir a independência do indivíduo, porém o declínio na habilidade para desempenhálas é um indicador precoce da redução da capacidade funcional (FONSECA; RIZZOTTO, 2008).

Seria importante que os instrumentos de avaliação funcional abordassem esse novo grupo ou que instrumentos específicos para esse construto fossem desenvolvidos, por serem atividades presentes no cotidiano das pessoas, sendo elas idosas ou não (KRAUSE; SILVA, 2006 apud FONSECA; RIZZOTTO, 2008).

Quanto à finalidade de uso dos instrumentos são poucos os artigos que a descrevem. Normalmente, essa descrição aparece nos instrumentos que são avaliativos, visto que os autores descrevem em seus estudos as alterações funcionais que os sujeitos apresentaram durante as pesquisas, demonstrando assim a sensibilidade do instrumento para qualquer alteração no desempenho funcional dos indivíduos.

Esse é um aspecto importante na descrição dos instrumentos, pois o foco e a finalidade da avaliação são importantes na escolha de um instrumento de avaliação.

Os instrumentos que em seus estudos não referem à finalidade, foram classificados nesta pesquisa através da definição de Tedesco (2002), sendo considerados instrumentos descritivos e avaliativos, como descrito anteriormente na introdução deste artigo.

A avaliação funcional é realizada através da observação e da mensuração da capacidade em realizar as atividades presentes em todos os grupos, pode ser compreendida como uma tentativa sistematizada de avaliar, de forma objetiva, os níveis de dependência e independência. Permite-nos detectar situações de risco, identificar áreas de disfunção e necessidades, monitorar o declínio funcional do idoso, estabelecer um plano de cuidado adequado às demandas assistenciais identificadas e identificar a necessidade de utilização de serviços especializados (FONSECA; RIZZOTTO, 2008).

No processo de avaliação de indivíduos com síndromes demências propõe-se a aplicação de um instrumento de avaliação funcional e um teste cognitivo, visto que essa combinação aumenta a sensibilidade e a especificidade para rastrear a doença (NITRINI et al., 2005).

Nitrini et al. (2005) sugerem para uma avaliação 
cognitiva global o Mini Exame do Estado Mental (FOLSTEIN et al., 1975) e para avaliação funcional o Questionário de Pfeffer (PFEFFER et al., 1982), IQCODE (JORM; JACOMB, 1989) ou Escala Bayer (LEHFELD et al., 1997).

Essa combinação aponta a importância de termos disponíveis, instrumentos de avaliação funcional, confiáveis e válidos, que abarquem o maior número de grupos de atividades visto que o desempenho funcional é amplo e heterogêneo.

Algumas limitações encontradas durante esse estudo foram: a dificuldade de acesso a todos os artigos levantados, dificuldade de acesso aos instrumentos de avaliação que não estavam disponíveis nos artigos produzidos e a carência de aporte teórico para a classificação dos instrumentos relacionada à sua finalidade de uso.

\section{CONCLUSÕES}

Foram selecionados 46 artigos e 20 teses, que utilizaram em seus estudos instrumentos de avaliação funcional. Quinze instrumentos foram identificados: 4 avaliam AIVD, 3 ABVD, 4 ABVD e AIVD, 3 ABVD, AIVD e Lazer e 1 ABVD e Lazer, evidenciando a falta de instrumentos que avaliem o grupo de atividades de trabalho, assim como a falta de uma avaliação que contemple todos os grupos de atividades.

Dos instrumentos identificados, 12 passaram pelo processo de adaptação transcultural, 9 apresentaram dados de confiabilidade e 5 de validade, sendo que um instrumento (MIF) apresentou validade para a população com lesões cerebrais e não com demência. O que demonstra a necessidade de estudos sobre a avaliação das propriedades psicométricas, uma vez que, 11 dos instrumentos selecionados não foram validados para o uso com idosos diagnosticados com demência, apesar de serem utilizados em estudos com essa população.

Por finalidade de uso 5 instrumentos foram classificados como avaliativos e 10 descritivos.

Os instrumentos de avaliação funcional utilizados no Brasil têm sua origem em outros países, evidenciando a falta de produções nacionais. O processo de adaptação transcultural tem proporcionado a utilização de instrumentos criados em outras línguas e adaptados a nossa cultural, porém a avaliação das propriedades psicométricas e a adaptação das notas de corte são dados tão importantes quando a adaptação.

A divisão dos instrumentos por finalidade, grupo de atividades e critérios do SAC, permite ao profissional a escolha de um instrumento adequado para a proposta de uso e contribui assim para um melhor atendimento e tratamento do paciente.

AGRADECIMENTO: Agradecemos à Fundação de Amparo à Pesquisa do Estado de São Paulo (FAPESP) pelo financiamento do projeto de Iniciação Científica (2008/09717-7).

CANON, M. B. F.; NOVELLI, M. M. P. C. Study of functional assessment tools in dementia commonly used in Brazil. Rev. Ter. Ocup. Univ. São Paulo, v. 23, n. 3, p. 253-262, set./dez. 2012.

\begin{abstract}
This research aimed to raise and identify the functional assessment tools used in dementia in Brazil. Data base were accessed from data base LILACS, PUBMED, Scielo and Portal Periódicos CAPES to arrange the studies related to functional assessment tools in dementia. Those instruments were selected and divided into activity groups and the purpose of use. This study identified if the instruments had gone through the process of cross-cultural adaptation, reliability and validity. Fortieth-sixth articles and 20 theses were selected and those ones used functional assessment instruments. Fifteen instruments were identified: 4 evaluate Instrumental Activities of Daily Living (IADL), 3 Basic Activities of Daily Living (ADL), 4 ADL and IADL, 3 ADL, IADL and leisure and $1 \mathrm{ADL}$ and leisure, highlighting the lack of instruments that assess work activities group, as well as the lack of an assessment that includes all of activities groups. Of the instruments identified, 12 instruments have cross-cultural adaptation, 9 of them analyzed the reliability and 5 the validity, 4 for use in dementia. For the purpose of use, 5 instruments were evaluative and 10 descriptors. This study demonstrated that the functional assessment tools used in Brazil have their origin in other countries, demonstrating the lack of national productions, in addition, the evaluation of psychometric properties is not being performed on all instruments adapted to our culture.
\end{abstract}

KEYWORDS: Aged; Dementia; Geriatric assessment. 


\section{REFERÊNCIAS}

AMERICAM PSYCHIATRIC ASSOCIATION (APA). Diagnostic and statistical manual of mental disorders. 4th ed. Washington, DC: APA, 2000.

ÁVILA, R.; BOtTinO, C. M. C.; CARVAlHO, I. A. M. et al. Neuropsychological rehabilitation of memory deficits and activities of daily living in patients with Alzheimer's disease: a pilot study. Braz. J. Med. Biol. Res., v. 37, p. 1721-1729, 2004.

BAHIA, V. S.; GOULART, M. T. C.; NOVELLI, M. M. P. C.; et al. Functional disability in Alzheimer s disease: a validation study of the Brazilian version of the Disability Assessment for Dementia (DAD-Br). Alzheimer Dis. Assoc. Disord., v. 13, p. 1-5, 2010.

BERTOLUCCI, P. H. F.; NITRINI, R. Proposta de uma versão brasileira para a escala ADCS-CGIG. Arq. Neuropsiquiatr., v. 61, p. 881-890, 2003.

BLESSED, G.; TOMLINSON, B. E.; ROTH, M. The association between quantitative measures of dementia and senile change in the cerebral gray matter of elderly subjects. Br. J. Psychiatry, v. 14, p. 797-811, 1968.

BUCKS, R. S.; ASHWORTH, D. L.; WILCOCK, G. K., et al. Assessment of activities of daily living in dementia: development of the Bristol activities of saily living scale. Age Ageing. v. 25, p. 113-120, 1996.

FOLQUITO, J. C.; BUSTAMANTE, S. E. Z.; BARROS, S. B.; et al. The Bayer - Activities of Daily Living Scale (B-ADL) in the differentiation between mild to moderate dementia and normal aging. Rev. Bras. Psiquiatr., v. 29, p. 350-353, 2007.

FONSECA, F. B.; RIZZOTTO, M. L. F. Construção de instrumento para avaliação sócio-funcional em idosos. Texto Contexto Enferm., v. 17, p. 365-373, 2008.

GIUSTI, E.; BEFI-LOPES, D. M. Tradução e adaptação transcultural de instrumentos estrangeiros para o Português Brasileiro (PB). Pró-Fono R. Atual. Cient., v. 20, p. 207-210, 2008.

INSTITUTO BRASILEIRO E GEOGRAFIA E ESTATÍSTICA (IBGE). Censo 2010. Rio de Janeiro, 2010. Disponível em: $<$ http://www.ibge.gov.br/home/estatistica/populacao/censo2010/ default_sinopse.shtm $>$. Acessado em: 10 maio 2011.

LANDIS, J. R.; KOCH, G. G. The measurement of observer agreement for categorical data. Biometrics, v. 33, n. 13, p.159174, 1977.

LEHFELD, H.; REISBERG, B.; FINKEEL, S.; et al. Informantrated Activities-of-Daily-Living (ADL) assessments: results of a study of 141 items in the U.S.A., Germany, Russia, and Greece from the International ADL Scale Development Project. Alzheimer Dis Assoc Disord. v. 11, p. 39-44, 1997.
LINO, V. T. S.; PEREIRA, S. R. M.; CAMACHO, L. A. B.; et al. Adaptação transcultural da Escala de Independência em Atividades da Vida Diária (Escala de Katz). Cad. Saúde Pública, v. 24, n. 1, p. 103-112, 2008.

MEDEIROS, M. E.; GUERRA, R. O. Translation, cultural adaptation and psychometric analysis of the Activities of Daily Living Questionnaire (ADLQ) for functional assessment of patients with Alzheimer's disease. Rev. Bras. Fisioter., v. 13, p. 257-266, 2009.

MELLO, M. A. R.; MANCINI, M. C. Avaliação das Atividades de Vida Diária e Controle Domiciliar. In: CAVALCANTI, A.; GALVÃO, C. Terapia ocupacional: fundamentação e prática. Rio de Janeiro: Guanabara Koogan, 2007. p.49-54.

MONTAÑO, M. B.; RAMOS, L. Validity of the Portuguese version of clinical dementia rating. Rev. Saúde Pública, v. 39, p. 1-5, 2005.

NITRINI, R.; CARAMELLI, P. H.; BOTTINO, C. M. C.; et al. Diagnóstico da doença de Alzheimer no Brasil: avaliação cognitiva e funcional. Recomendações do Departamento de Científico de Neurologia Cognitiva e do Envelhecimento da Academia Brasileira de Neurologia. Arq Neuropsiquiatr., v. 63, p. 720-727, 2005.

PAPALEO NETTO, M. Processo de envelhecimento e longevidade. In: PAPALEO NETTO, M. Tratado de Gerontologia. São Paulo: Atheneu, 2007. p.3-14.

NOVELLI, M. M. P. C.; NITRINI, R.; CARAMELLI, P. Validation of the Brazilian version of the quality of life scale for patients with Alzheimer's disease and their caregivers (QOL-AD). Aging Mental Health, v. 14, n. 5, p. 1-8, 2010.

PEREIRA, L. S. M.; MARRA, T. A.; FARIA, C. D. C. M. et al. Adaptação tanscultural e análise de confiabilidadedo Southampton Assessment of Mobility para avaliar a mobilidadede idosos brasileiros com demência. Cad. Saúde Pública, v. 22, n. 10, p. 2085-2095, 2006.

PEREIRA, F. S.; OLIVEIRA, A. M.; DINIZ, B. S.; et al. Crosscultural adaptation, Reliability and validity of the DAFS-R in a Sample of Brazilian Older Adults. Arch. Clin. Neuropsychol.. v. 25, n. 4, p. 335-343, 2010.

PFEFFER, R. I.; KUROSAKI, T. T.; HARRAH, C. H. Jr.; et al. Measurement of functional activities in older adults in the community. J. Gerontol., v. 37, p. 323-329, 1982.

POLGAR, J. M. Avaliação Crítica. In: CREPEAU, E. B.; NEISTADT, M. E. Willard and Spackman: terapia ocupacional. Rio de Janeiro: Guanabara Koogan, 2002. p.153-166.

RIBERTO, M.; MIYAZAKI, M. H.; JUCÁ, S. S. H.; et al. Validação da versão brasileira da medida de independência funcional. Acta Fisiatr., v. 11, p. 72-76, 2004. 
CANON, M. B. F. et al. Estudo dos instrumentos Rev. Ter. Ocup. Univ. São Paulo, v. 23, n. 3, p. 253-62, set./dez. 2012.

SANCHEZ, M. A. S.; LOURENÇO, R. A. Informant Questionnaire on Cognitive Decline in the Elderly (IQCODE): adaptação transcultural para uso no Brasil. Cad. Saúde Pública, v. 25, p.1455-1465, 2009.

SANTOS, R. L.; VIRTUOSO Jr., J. S. Confiabilidade da versão brasileira da Escala de Atividades Instrumentais da Vida Diária. RBPS. v. 21, p. 290-296, 2008.

SCIENTIFIC ADVISORY COMMITTEE OF THE MEDIACL OUTCOMES TRUST (SAC). Instrument Rev. Criteria, v.3, n. 4, 1995.

Recebido para publicação: 16/12/2011

Aceito para publicação: 19/08/2012
SCIENTIFIC ADVISORY COMMITTEE OF THE MEDIACL OUTCOMES TRUST (SAC). Assessing health status and qualityof-life instruments: Attributes and review criteria. Quality Life Res., v. 11, p. 193-205, 2002.

TEDESCO, S. O desenvolvimento e estudo de instrumentos de avaliação em Terapia Ocupacional. Cad. Centro Universitário S. Camilo, v. 8, p. 17-26, 2002.

VASCONCELOS, L. G.; BRUCKI, S. M.; BUENO, O. F. A. Cognitive and functional dementia assessement tools: Review of Brazilian literature. Dementia Neuropsychol., v. 1, p. 18-23, 2007. 